\title{
ERICH SCHWEIGHOFER - EIN PIONIER DER RECHTSINFORMATIKFORSCHUNG ZUR VERANTWORTUNGSVOLLEN DIGITALISIERUNG
}

\author{
A Min Tjoa
}

Erich Schweighofer kann weltweit als einer der renommiertesten Wegbereiter der juristischen Wissensrepräsentation mit Mitteln der Informatik (insbesondere auf dem Gebiet der Artificial Intelligence und Information Retrieval) angesehen werden.

Bereits vor etwa 25 Jahren hat er als einer der ersten Rechtswissenschaftler Methoden aus dem Gebiet der Neuronalen Netzwerke zur automatischen Generierung von Begriffsausprägungen, Dokumentbeschreibungen und Hypertextlinks sehr erfolgreich eingesetzt.

Seit Anbeginn seiner wissenschaftlichen Tätigkeit als Rechtsinformatiker war es ihm immer ein Anliegen, den wichtigen Schritt zu vollziehen, «das Recht als Informationssystem mit rechtlich formalisierten Informationsprozessen» zu betrachten. Dieser Schritt von der Anwendung von Methoden der Informatik für rechtlich formalisierte Prozesse zur «Darstellung des Rechts als Informationssystem» kann als übergeordnetes Motto seines wissenschaftlichen Oeuvres betrachtet werden. Nicht zuletzt ist diese Betrachtungsweise seiner Ausbildung als Wirtschaftsinformatiker zu verdanken, wo man den Paradigmenwechsel von der Anwendung von Informationssystemen in den betrieblichen Informationsprozessen zur Darstellung des Betriebes als Informationssystem vollzogen hat.

Als ausgewiesener Experte auf dem Gebiet der Informatik bzw. Wirtschaftsinformatik und gleichzeitig als Rechtswissenschaftler war es immer Erich Schweighofers Bestreben, als überzeugter Rechtsinformatiker neueste wissenschaftliche Erkenntnisse und 
Innovationen auf dem Gebiet der Informatik für rechtsinformatische Forschungsfragen erfolgreich zu nutzen. Umgekehrt hat die Informatikcommunity in Österreich von seiner transdisziplinären Herangehensweise sehr viel profitiert.

Seine Tätigkeit im hochkarätig besetzen Rat für Robotik und Künstliche Intelligenz ist von höchster Relevanz für die ethische Weiterentwicklung auf dem Gebiet der Artificial Intelligence und Industrie 4.0 mit all seinen Konsequenzen für den Schutz der Privatheit und Menschenwürde. Für alle Informatiker im deutschen Sprachraum und in der EU bilden die Erkenntnisse dieses Spitzengremiums eine erste Richtschnur zur Bewertung von Risiken und Chancen und für ethisches Handeln im Rahmen der täglichen Arbeit in Forschung und Industrie. Die Mitarbeit eines Rechtsinformatikers mit dem Profil des Jubilars ist ein «wahrer Segen» für eine möglichst umfassende Formulierung der Forcierung des ethischen Einsatzes von Robotik, Autonomen Systemen und Künstlicher Intelligenz. Schweighofer hilft uns Informatikern durch diese Arbeit, «Rahmenbedingungen zu gestalten, die bestimmen, in welcher Welt wir künftig leben wollen.»

Sein Wirken als Forscher und Lehrender am Institut für Europarecht, Internationales Recht und Rechtsvergleichung an der Universität Wien und seine langjährige Tätigkeit bei der EU-Kommission in Brüssel sind genau die Ingredienzen für eine Wissensansammlung, die idealer Weise benötigt werden, um das «Recht als Informationssystem mit rechtlich formalisierten Informationsprozessen zu betrachten.»

Der Erfolg des Internationalen Rechtsinformatik Symposion IRIS ist engstens mit dem Namen Erich Schweighofer verknüpft. Sein Beitrag bei der fortwährenden Entwicklung dieses Symposiums seit seiner Entstehung spiegelt in vielerlei Hinsicht auch die Breite seines Interessensspektrums wider. IRIS macht die vermehrte Durchdringung der Rechtsinformatik durch andere Wissensdisziplinen sehr deutlich. Das Motto von IRIS 2020, wo unser Jubilar Programmvorsitzender ist, lautet «Verantwortungsvolle Digitalisierung». Diese Maxime könnte auch für das ganze wissenschaftliche Wirken von Erich Schweighofer stehen. Erich war stets bestrebt, den Gestaltungsraum neuester technologischer Entwicklungen so zu gestalten, dass rechtlich ein Interessenausgleich gewährleistet ist, wo negative Auswirkungen von vornhinein vermieden werden. 
Als Vorsitzender und Gestalter des Arbeitskreises Rechtsinformatik der Österreichischen Computer Gesellschaft hat Erich in seiner seriösen und stets bescheidenen Arbeitsweise sehr viel dazu beigetragen, dass ethische Grundsätze in Österreich den absoluten Vorrang haben sollen bei der Lösung von rechtsinformatischen Fragen.

Für mich war es seit Anbeginn unserer akademischen Beziehung und Freundschaft stets ein intellektueller Genuss, in den vielen Diskussionen mit Erich seine oft sehr langen Argumentationsketten nachzuvollziehen. Dieser Nachvollzug seiner Gedanken weckt bei mir fast immer Assoziationen zur formal mathematischen Beweisführung.

Wir von der Scientific Community sind alle Erich sehr dankbar, dass er immer zur Stelle war, wenn man seine Unterstützung für ein sinnvolles Projektvorhaben braucht, wo seine Kompetenz unabdingbar notwendig ist.

Ich wünsche Erich für die nächsten Jahrzehnten weiterhin so viel wissenschaftlichen Erfolg und vor allem den gesellschaftlichen «Impact» für sein Wirken im Dienste einer «Verantwortungsvollen Digitalisierung».

Ad multos annos! 
